\title{
Low-voltage Power Line Broadband Communication System
}

\author{
Du Peidong, Wang Weizhou, Liu Fuchao, Zheng Jingjing
}

State Grid Gansu Electric Power Research Institute, Lanzhou, 730050, China

peidong9@163.com

Keywords: Power Line Communication, Power Line Modem, Power Line Communication Network.

\begin{abstract}
The power line communication (PLC) technology can make full use of the most universal power line network resources with high construction speed, less investment, and no wiring inside the house. The users could achieve high-speed Internet access through power plug seats of each room, and finally realize "mobility with cable" with incomparable advantage which other access ways don't possess, and it could also solve the bottleneck of popularization of Internet. And it is considered as the "last one hundred meters" problem of broadband access by industry insiders. After realizing point-to-point communication by using power line modem, we use power line communication network to realize broadband power line communication system network. The ultimate goal of networking is to choose appropriate network topology structure so as to ensure that the communication network is stable with reliable performance.
\end{abstract}

\section{Power Line Network Unit}

Basic Network Unit. For the realization communications services by using power supply network, some of the basic units are indispensable. The main function these basic units lie in complete the generation and transformation of signals making the signals can be transmitted and received through the power line. Each PLC broadband access network contains two types of equipment: PLC modems (PLC Modem) and PLC Master/Base stations (PLC Master/Base Station).

PLC Base stations (or Master stations) means connect the PLC access to its backbone networks, it realized the connection between the power transmission medium and backbone telecommunication network. However, base station does not directly connect user terminal equipment, but to provide all kinds of network communication interfaces. That includes xDSL, Synchronous Digital series used to connect to the network (SDH, Synchronous Digital Hierarch), WLL used in the wireless appliances etc.

Repeaters. At present the frequency range has been or will soon be used by the management institutions of PLC technology is limited (the highest frequency is approximately $30 \mathrm{MHZ}$ ). Therefore, with the increase of the use of different frequency ranges, the number of public frequency bands will be split to many very narrow frequency parts, which will significantly reduce the capacity of the network. Therefore, a good PLC access frequency division should be able to use as few frequencies ranges as possible. Using Repeaters can effectively prolong the distance network coverage that the PLC technology can provide, but also could raise the costs of the network due to the increase of the cost in the equipment and its installation. As a result, the number of the repeaters used in PLC access network should be as few as possible.

PLC Gateway. By the following two kinds of method, PLC users could access to the network through the wall outlet PLC access mode: connected directly or through a gateway to connect indirectly.

Gateway users divide the PLC access network and indoor PLC network into two parts, which will also be responsible for the transmission of signal in access and indoor application under different frequencies. Such a gateway is usually located in the neighboring houses of electric meter units. Besides, the PLC gateway could at the same time ensure the realization of the logical network level access and the identification of interior areas. As a result, PLC modems used for indoor network connection could realize the internal communication within without letting the information access into network. In this case, the gateway function of a PLC is just like a logic base station 
controlling the indoor PLC network, which could, on the one hand, coordinate the internal communications between PLC modems, on the other hand, also could coordinate the access network communication between internal the equipments and PLC.

\section{Connect with the Core Network Connection}

PLC access system can be used to override the so-called "the last kilometer" telecommunications access areas. Which means, under the condition that there is low-voltage distribution network, the application of PLC technology can solve the problem of the last a few hundred meters access network construction. On the other hand, PLC access network through the communication distribution network to realize the connection among backbone networks.

The application of PLC technology can save the cost of laying new telecom network. However, PLC access network must through the backbone network to achieve the connection between the wan net, which would increase the cost to a certain degree. From that point of view, the cost of the realization of the PLC backbone network in should be as low as possible, only by this way, the relative competitiveness of PLC network over other access technologies could be maintained.

The Communication Technologies Used in PLC Distribution Network. The mature communication technologies that have already been used in the application field can be used at very low cost to achieve the connection between PLC access network and backbone network. Some of the transformer units have been connected to the maintenance network through a standard communication cable (copper wire). At the beginning, these connections are used to realize the function of remote control or the communication among power supply network control center, maintenance personnel and equipment. However, through DSL technologies, these cables can realize the connection between the PLC network and the backbone network.

While applying communication technologies to PLC distribution network, it must be ensured that the technology could support the entire business transmission provided by the PLC access network. In addition, the backbone network of PLC should not become the bottleneck of the communication between PLC general users and the backbone networks. Therefore, technologies used in backbone network must be able to provide enough transmission capacity (data transfer rate) and ensure to meet the needs of different Quality of Service s (QoS, Quality of Service).

The Topological Structure of Distribution Network. The main network topology is a technical scheme that could allow the network distributed within appropriate area at a low cost. Cost factor, however, is not the only standard to choose a distribution network topology structure, another very important factor is the network reliability under the condition of network failure. In the main topology structure, if there is a fault between two PLC access network connecting links, all access network link built after the fault will lost connection with a WAN net. Therefore, while considering the PLC network topology structure, the topology of the network should be taken into consideration.

Finally, PLC distribution network topology structure can also make the above mentioned three kinds of basic network structures cooperate with each other. However, while selecting network topology structure, the following factors should be taken into consideration:

(1) The communication technology in use determines the special network structure;

(2) The transmission mediums currently used in applications field;

(3) Realizing the feasibility of the reliable distribution network;

(4) The geographical structure, the PLC access network the distribution, the locations of local exchange stations.

The Management of the PLC Access Network. The management of PLC access network includes configuring and reconfiguring the network unit according to the current state of each network (base station, a modem, Repeaters and gateway). These management functions can be achieved by the local base station or gateway, and they could also be realized by the management center through remote control. Local management functions can be completed automatically without the participation of relevant personnel. Remote management, on the other hand, can be achieved through the automatic control and artificial execution these two ways. The transmission of 
access network management information can be carried out by the PLC distribution network, so as to avoid the communication installation costs of communication system specially used for the transmission of management information. An effective management plan is to put management and maintenance function as much as possible to the PLC access network base station and gateway to complete. However, carrying on management functions will increase the equipment cost of PLC network unit. As a result, there is an optimization problem of how to reasonably divide management function between network unit and the control center.

\section{Power Line Broadband Network Mode}

The structure of domestic low-voltage distribution network is complex, different floors and construction period of the building has a different distribution network structure, so the access solutions have not yet to be unified. The structure of PLC network is flexible, which allows reasonable changes based on the number of users. According to the characteristics of the distribution network line, building structures and users, solutions can be divided into the following three kinds of situations: high-rise residential building, low-rise residential building and commercial office building.

The Network Structures of High-Rise Residential Buildings. Terminal PLC office in the distribution room of the basement uses the layer of the same power line or adjacent layers of users as a shared area, sharing the same terminal PLC. If there are fewer users, multiple shared areas could be joined onto the same device. Such as, dividing the users according to the three phase line. Assume that A line of users share A power line, even let all the users in the building share the same power line, thus we could assure users maintain relatively high utilization rate while assuring that the users could use the Internet bandwidth. After the number of users, it could facilitate the division of the shared areas while not impact on the original user. Equipment of client-PLC MODEM isolates data signals from power line and uses USB or RJ45 interfaces to connect with the users' computers to ensure the end-to-end 2 MBPS bandwidth run reasonably. Multiple PLC Modems could constitute a small local area network (LAN). The plan is mainly for high-rise residential buildings where the users concentrated and there is higher network use rate, this plan can be combined with various applications, such as remote meter reading, domestic automation.

Commercial Office Network form Mode. Commercial office building is mainly for small and medium enterprises, and these users demand lower network bandwidth and security needs. PLC broadband access can meet the requirements of these kinds of users.

Office access adopts the FTTB + Ethernet + PLC plan, and the fiber is for each building, and through the optical fiber network, the fiber is connected with PLC network. Install a switch or a hub as the core exchange equipment of the building. On each floor, to distribute integrated wiring we assure that $100 \mathrm{M}$ bandwidth shall be installed to the floor. On the distribution room floor, install a terminal PLC, and divide the shared areas according to the enterprise users' need. Users with more interfaces could enjoy a PLC device all by themselves, and users with fewer interfaces could share the PLC equipment.

Low-Rise Residential Area Network Mode. Building structure features: each of the underlying residential building has 5 to 6 layers, and it is divided into multiple units with each unit having its own staircase, there are 60-80 residents in the 2-4 floors of each layer, and the some larger ones have 100 households.

Low voltage power distribution network structure: A distribution transformer is responsible for the 5 or 6 buildings, from bottom to the top, each unit has a power line. There is a electric meter on each floor, and on the first floor, there is the total meter for all users in the unit.

Networking scheme: Apply the FTTB + PLC or optical fiber to the transformer + PLC program, the community could be connected to the MAN (metropolitan area network) through optical fiber.

For the underlying residential buildings, in which residents are relatively concentrated and the Internet needed fewer. On the basis of physical distribution network structure division, a unit set should be placed with a terminal PLC, thus the users could share a same power line. While there are fewer users, several units or even a few buildings can share a office terminal device. While the 
number of users increased, the shared area should be distributed flexibly again according to the distribution of users.

Through USB, RJ45 interfaces, Client PLC Modem could be connected to users' computers to ensure the end-to-end's 2 MBPS transmission rate, and multiple PLC Modems form a small local area network (LAN).

\section{References}

[1] Radford D. Spread-Spectrum Data Leap Through AC Power Wiring • IEEE Spectrum. 1996

[2] Manfred Zimmermann. Klaus Dostert. Analysis of the Broadband Noise Scenario in Powerline networks. Proceedings of the 4th International Symposium on Powerline Communications and its Applications, Linerick, Ireland, April 5th-7th,2000:131-138

[3] Liu D. Wide Band AC PowerLine Chsracter+-tion. IEEE Tran on Consumer Electronics, 1999, 45(4): 89-83

[4] H. Philips, Development of a Statistical Model for Powerline Communications Channels, Proceeding of the 7"' International Symposium on Powerline Communications and its Applications, Limerick, Ireland, 2000. 